\title{
The Next Generation of IR Spectroscopy: EC-QCL-Based Mid-IR Transmission Spectroscopy of Proteins with Balanced Detection
}

\author{
Christopher K. Akhgar, Georg Ramer, Mateusz Żbik, Artur Trajnerowicz, Jarosław Pawluczyk, \\ Andreas Schwaighofer,* and Bernhard Lendl*
}

Cite This: Anal. Chem. 2020, 92, 9901-9907

Read Online

\section{ACCESS | Llll Metrics \& More | 回 Article Recommendations | (s) Supporting Information}

ABSTRACT: We report a mid-IR transmission setup for the analysis of the protein amide I and amide II band in aqueous solutions that achieves a limit of detection as low as $0.0025 \mathrm{mg}$ $\mathrm{mL}^{-1}$ (outperforming our previous results and other state-of-the-art mid-IR-based techniques by almost an order of magnitude). This large improvement is made possible by combining the latestgeneration external cavity-quantum cascade laser (EC-QCL) operated at room temperature with an optimized double-beam optical setup that adjusts the path length $(26 \mu \mathrm{m})$ to ensure robust sample handling. For minimizing the noise introduced by the highintensity laser light source, a thermoelectrically cooled mercury cadmium telluride balanced detection module was employed. In this
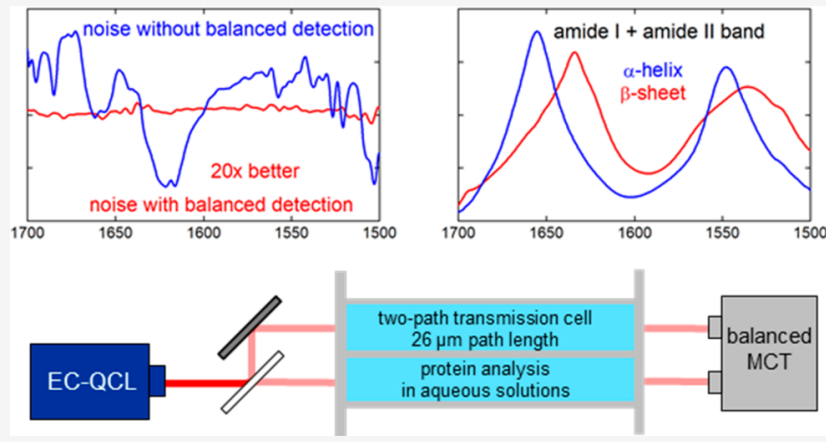
way, noise levels better by a factor of up to 20 were achieved compared with single-channel measurements. Characteristic spectral features of proteins with different secondary structures were successfully identified at concentrations as low as $0.1 \mathrm{mg} \mathrm{mL}^{-1}$. Furthermore, a highly linear response was demonstrated for concentrations between 0.05 and $10 \mathrm{mg} \mathrm{mL}^{-1}$. The total acquisition time of the setup can be adapted to fulfill the required sensitivity of the protein measurements and to ensure maximum flexibility for future applications. The presented setup combines high sensitivity, large optical path lengths, and short measurement times and thus outperforms previous research type EC-QCL setups as well as commercially available instruments. This opens a wide range of future applications including protein-ligand interaction studies as well as qualitative and quantitative analyses of proteins in complex matrices such as those found in up- and downstream bioprocess monitoring and similar challenging applications which can not be readily met by conventional FT-IR spectroscopy.

$\mathrm{T}$ he mid-infrared (mid-IR) region $\left(4000-400 \mathrm{~cm}^{-1}\right.$ ) provides detailed information about the specific rotational-vibrational transitions of molecules. Polypeptides and proteins show characteristic absorption bands in the IR spectrum that are referred to as amide bands. The most prominent band for secondary structure analysis is the amide I band $\left(1700-1600 \mathrm{~cm}^{-1}\right){ }^{1}$ Additionally, the amide II band $\left(1600-1500 \mathrm{~cm}^{-1}\right)$ has been recognized to be sensitive to secondary structure. ${ }^{2}$ The position and shape of the amide bands show sensitivity to secondary structure due to changes in the interaction of the $\mathrm{C}=\mathrm{O}$ and $\mathrm{N}-\mathrm{H}$ groups of the amide bond induced by different arrangements of hydrogen bonding, dipole-dipole interactions, and geometric orientations in the $\alpha$-helices, $\beta$-sheets, turns, and random coil structures. ${ }^{3}$ For quantification of the individual secondary structure components, curve fitting ${ }^{4}$ or chemometric approaches such as partial least-squares ${ }^{5}$ or multivariate curve resolutions-alternating least-squares (MCR-ALS) ${ }^{6}$ can be applied to the amide I region. However, it has been shown that additional and more comprehensive information can be gained by combined analysis of the amide I and II bands, particularly with chemometric analysis. ${ }^{7-9}$
Fourier transform infrared (FT-IR) spectroscopy, the most widespread type of mid-IR spectroscopy is a powerful and wellestablished method for quantitative and qualitative analysis of proteins. ${ }^{10,11}$ FT-IR instruments are commonly equipped with thermal light sources such as Globars that emit a broadband but low-power and constant radiation. For samples with highly absorbing matrix components, the low emission intensity of the light source can lead to limitations of the measurements. A pronounced challenge in mid-IR spectroscopy of proteins is the strong absorption of the $\mathrm{HOH}$-bending band of water near $1643 \mathrm{~cm}^{-1}$ that overlaps with the amide I region of proteins. For conventional methods, the optical path length is restricted to $<10 \mu \mathrm{m}$, in order to avoid total IR absorption. ${ }^{1,12}$ These low path lengths lead to impaired sensitivity and limited robustness

Received: April 1, 2020

Accepted: June 29, 2020

Published: June 29, 2020 
because of the low-intensity IR bands and likely clogging of the cell when measuring a sequence of samples.

More than two decades ago, quantum cascade lasers (QCLs) were introduced as a polarized, coherent and high power light source in the mid-IR region. ${ }^{13}$ They combine stable operation at room temperature and increased spectral power densities (SPDs) by a factor of $10^{4}$ and more compared with thermal light sources. ${ }^{14}$ In combination with an external diffraction grating for tuning in a so-called external cavity (EC) design, EC-QCLs offer broadband spectral tuning in excess of several hundred wavenumbers, making them favorable for the analysis of liquid samples. It has been shown that the high available emission power of QCLs enable mid-IR transmission analysis of proteins at an optical path length 4-5 times larger than with conventional FT-IR spectroscopy. ${ }^{15-23}$

While the high power of EC-QCLs is an advantage over FTIR spectroscopy, they suffer from noise that FT-IR spectroscopy is immune to, including (1) pulse-to-pulse intensity fluctuations around $2 \%$ and (2) wavelength reproducibility issues due to their wavelength tuning mechanism. Furthermore, EC-QCLs have an SPD that is defined by the wavelength-dependent gain of the $\mathrm{QC}$ gain material. Hence, EC-QCLs can easily saturate a detector in one part of the spectrum while dropping below the noise limit at another. For FT-IR spectrometers, wavelength stability is given because of Connes' advantage, and the noise level of the thermal light sources can usually be neglected compared with the detector noise. High-intensity laser light sources however contribute to the overall noise level, especially when operated in pulsed mode, because of pulse-to-pulse intensity fluctuations. ${ }^{24,25}$

The challenge in building a high-sensitivity EC-QCL spectroscopy setup is thus to make the most of the advantages of the source while at the same time removing or handling its current disadvantages.

For example, inconsistent wavelength tuning behavior of the first-generation EC-QCL was overcome by a data processing routine based on correlation optimized warping (COW) and filtering to correct shifts in the fine structure throughout the spectrum. ${ }^{15}$ For second generation EC-QCLs, optimized motion mechanics and triggering routines of the external cavity were introduced. Moreover, a higher tuning range and increased scan speed enabled protein measurements in the amide I and amide II region at lower concentrations within shorter measurement times. Significant improvements were made; nevertheless, a mathematical routine was still needed to identify shifted scans which had to be removed prior to signal averaging for achieving best possible performance. Furthermore, the remaining challenges of a highly uneven SPD and large pulse-to-pulse fluctuations were also limiting the potential of EC-QCL transmission-absorption spectroscopy. ${ }^{19}$ Here we present an optical design that overcomes some of these challenges to perform EC-QCL protein spectroscopy at unprecedented sensitivity.

The uneven SPD is counteracted by a set of optical attenuators that are matched to the sample matrix absorption and the emission spectrum of the QCL. To suppress laser intensity noise (thermal drifts and $1 / \mathrm{f}$ ), balanced detection was proposed by Hobbs. ${ }^{26}$ Here, the laser beam is split into two beams of equal intensities called the signal and reference beam. Only one of the beams interacts with the sample, but both are detected on matched detectors. The resulting voltages are subtracted from each other to yield the "balanced output". Any change in intensity that afflicts both beams is thus suppressed in the balanced output. Previously, balanced detection was successfully applied for the reduction of the noise introduced by different types of QCL light sources for gas-phase analysis $^{27,28}$ and to increase the sensitivity of an EC-QCLbased detector for size-exclusion chromatography (SEC).$^{29}$ In that last implementation, a low dead volume fiber optic-flow cell was used, ${ }^{30,31}$ and balancing was achieved by attenuation of the reference beam by means of a variable aperture to adjust for uneven SPD of the employed EC-QCL.

We demonstrate highly sensitive protein spectroscopy in $\mathrm{H}_{2} \mathrm{O}$ using an optimized EC-QCL liquid spectroscopy setup based on improved balanced detection and adjusted SPD. The performance of the system is characterized and benchmarked against previous EC-QCL-based designs showing that it outperforms not only previous EC-QCL setups but also high-end FT-IR and commercial laser-based systems.

\section{EXPERIMENTAL SECTION}

Reagents and Samples. Hemoglobin from bovine blood, concanavalin A from jack bean, and $\gamma$-globulins from bovine blood $(\geq 97 \%)$ were purchased from Sigma-Aldrich (Steinheim, Germany). Proper amounts of protein powder were dissolved in water. Ultrapure water (resistivity: $18 \mathrm{M} \Omega \mathrm{cm}$ ) was purified with a Milli-Q system from Millipore (Bedford, MA).

Balanced Detection Module. The balanced detection module developed by Vigo (Vigo System S.A., Poland), uses two individual thermoelectrically cooled mid-IR mercury cadmium telluride (MCT) detectors, operated at $-73{ }^{\circ} \mathrm{C}$ with a $0.5 \times 0.5 \mathrm{~mm}$ element size and a detectivity of $D^{*}=$ $1.59 \times 10^{10} / 1.36 \times 10^{10} \mathrm{~cm} \mathrm{~Hz}^{0.5} \mathrm{~W}^{-1}$ at $8 \mu \mathrm{m}$ at $200 \mathrm{kHz}$. These two detectors are precisely matched regarding their transimpedance, voltage responsivity, and detectivity to achieve a high common mode rejection ratio (CMRR). The working principle and electronic circuit are presented in Figure $S 1$ in the Supporting Information. In order to compensate for dark currents (in the order of tens of $\mu \mathrm{A}$ ) that are present in MCT structures, manual compensation subcircuits were implemented. The detectors are connected to two identical amplifier channels (signal and reference). Each channel consists of a transimpedance amplifier followed by a fixed gain voltage amplifier. External instrumentation is connected via buffered outputs. To generate "balanced output", signal and reference channels are combined in the subtractor block, where an analogue subtractor generates the difference signal (reference channel-sample channel). To achieve high attenuation of common mode changes in the optical signal, the received power in the signal and reference beam should be made as similar as possible $\left(I_{\text {signal }} / I_{\text {reference }} \approx 1: 1\right)$. Any power difference in the optical signals (differential mode signal) is present at the balanced output, whereas unwanted common mode signals are attenuated.

Experimental Setup for QCL-IR Measurements. Figure 1 shows the schematic of the experimental laser-based setup. The applied EC-QCL (Hedgehog, Daylight Solutions Inc., San Diego U.S.A.) was operated with a laser current of $650 \mathrm{~mA}$, a repetition rate of $1 \mathrm{MHz}$ and a pulse width of $200 \mathrm{~ns}$ (duty cycle of $20 \%$ ). The operating temperature of the water-cooled laser head was set to $19{ }^{\circ} \mathrm{C}$. Spectra were recorded between 1730 and $1470 \mathrm{~cm}^{-1}$, using a scan speed of $3600 \mathrm{~cm}^{-1} \mathrm{~s}^{-1}$. The QCL emission spectrum was amended with optical components in order to avoid detector saturation in certain spectral regions (see Adaptation of Laser Emission and Detector Characteristics for details). A wire mesh was used to reduce the 


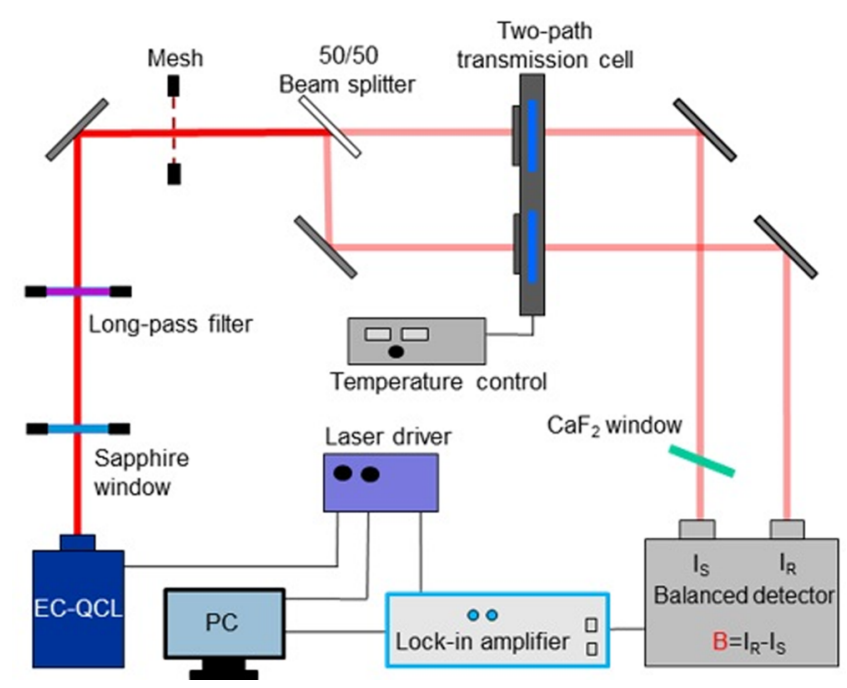

Figure 1. Schematic of the two-path QCL-based mid-IR transmission setup for protein measurements in aqueous solutions.

IR-light intensity equally across the entire scan range. In order to adjust the maximum laser intensity to the dynamic range of the detector, ${ }^{19}$ a short-pass filter (wedged sapphire window, $2.5 \mathrm{~mm}$ thickness) and a silicon-based long-pass filter were employed to selectively lower the laser intensity in the amide II region and in the high wavenumber range of the amide I band, respectively, leading to a pulsed average power of approximately $5.4 \mathrm{~mW}$ at $1650 \mathrm{~cm}^{-1}$. Then, the laser light was divided into two beams by a nominal 50:50 beam splitter (Thorlabs BSW510). The two beams were directed into a custom-built two-path $\mathrm{CaF}_{2}$ transmission flow cell with optical paths of 26 $\mu \mathrm{m}$. The temperature of the transmission cell was set to $20^{\circ} \mathrm{C}$ by using a thermoelectric-cooling temperature controller (Meerstetter Engineering GmbH, Rubigen, Switzerland). The thermoelectrically cooled balanced MCT detector module was used to detect the light intensities of the two beams. $\mathrm{A} \mathrm{CaF}_{2}$ window was positioned in front of one of the detecting elements to specifically attenuate the intensity of one laser beam to match the signal intensities on the two detector elements, as outlined below (see Balanced Detection for
Reduction of Spectral Noise for details). The optical setup was encased with an acrylic glass housing and continuously flushed with dry air to decrease the influence of water vapor from the atmosphere.

The measured output signals (signal, reference, balanced) were processed and digitized by a lock-in amplifier MFLI (Zurich Instruments, Zurich, Switzerland) with an extended cutoff range of $5 \mathrm{MHz}$ and up to four demodulators (F5M and MD addons). The lock-in demodulator frequency was referenced to the laser repetition rate. As the balanced signal can have positive and negative amplitude, the in-phase (X) signal was recorded. Single-beam spectra were recorded during a tuning time of approximately $72 \mathrm{~ms}$ and consisted of 5401 data points. For protein measurements, 300 scans were averaged, leading to a total acquisition time of $45 \mathrm{~s}$. Disposable syringes were used to manually inject approximately $500 \mu \mathrm{L}$ of solution into the flow cell. Spectral resolutions of 0.5 and 2.6 $\mathrm{cm}^{-1}$ were determined for nonfiltered and filtered spectra, by comparing the bandwidth of water vapor spectra to FT-IR spectra acquired at different resolutions. The wavenumber scale of the measured spectra was obtained by calibration against the absorption bands of water vapor. A graphical user interface provided by Daylight Solutions was used to control the laser. Data acquisition was performed using in-house developed Python script (Python 3.7) to access the MFLI API.

Processing of QCL Data. A detailed description of the data preprocessing routine can be found elsewhere. ${ }^{19}$ Briefly, the performed preprocessing steps included smoothing, similarity index evaluation, scan averaging, fast Fourier transform (FFT) filtering and calculation of the final absorbance spectra. For the presented setup, scans with a similarity index below 0.94 were removed. This value showed optimal results for balanced detection single channel spectra. An FFT filter with a cutoff frequency of $1500 \mathrm{~Hz}$ was applied to the single beam spectra. Before recording a measurement run an initial single channel signal $\left(I_{\text {ref, }, 0}\right)$ was recorded and stored. This single channel was then added to both background $\left(I_{\text {bal,bg }}\right)$ and measurement $\left(I_{\text {bal,smp }}\right)$ balance signal before calculating absorption:

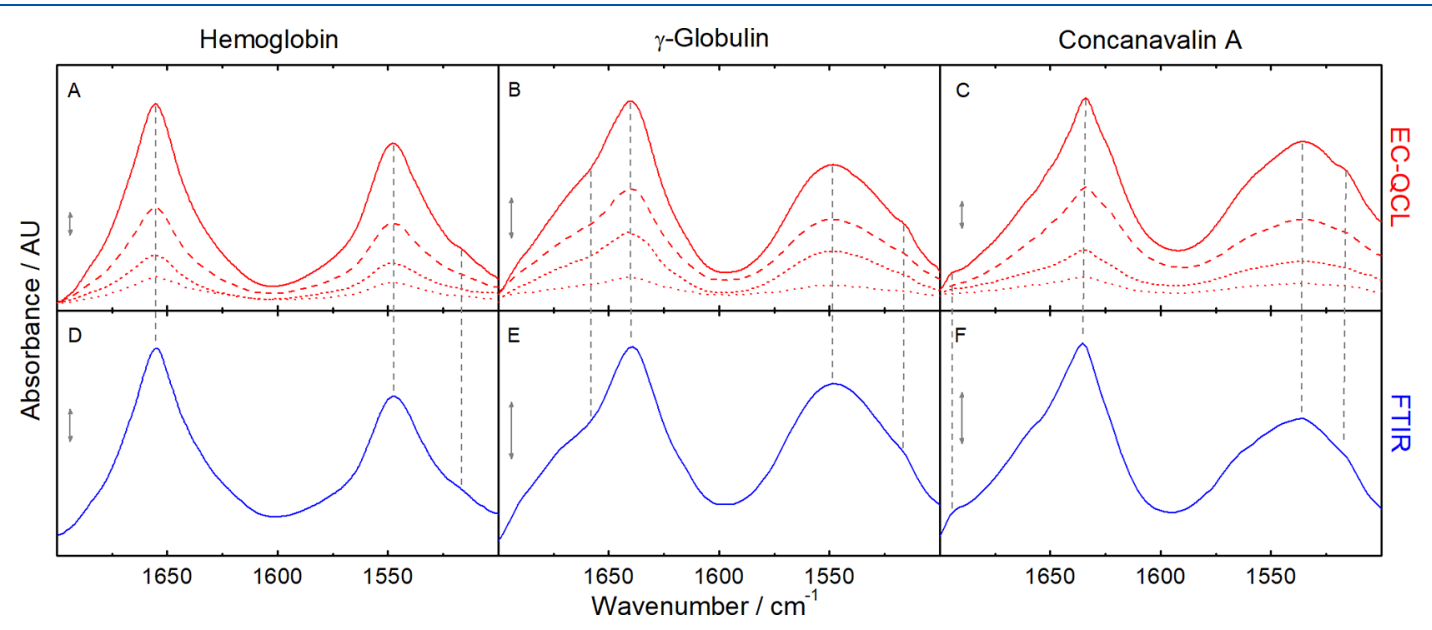

Figure 2. Mid-IR absorbance spectra of 1 (red solid line), 0.5 (red dashed line), 0.25 (red short dashed line), and $0.1 \mathrm{mg} \mathrm{mL}^{-1}$ (red dotted line) protein solutions recorded with the EC-QCL setup (A-C) and $20 \mathrm{mg} \mathrm{mL}^{-1}$ (blue solid line) protein solutions obtained by high-end FT-IR spectroscopy $(\mathrm{D}-\mathrm{F})$. Gray dashed lines highlight the high congruence of the spectral features between the mid-IR spectra acquired by EC-QCL and FT-IR spectroscopy. Gray double-headed arrows indicate the absorbance of $1 \mathrm{mAU}$ for QCL-IR and $10 \mathrm{mAU}$ for FT-IR spectra. 


$$
A=\log \left(\frac{I_{\text {ref }, 0}+I_{\text {bal, bg }}}{I_{\text {ref }, 0}+I_{\text {bal }, \text { smp }}}\right)
$$

To compare the protein absorbance spectra acquired by FT-IR spectroscopy $\left(\mathbf{s}_{1}\right)$ and EC-QCL $\left(\mathbf{s}_{2}\right)$ quantitatively, the degree of spectral overlap $\left(s_{12}\right)$ was calculated by using the following expression: ${ }^{32}$

$$
s_{12}=\frac{\left\|\mathbf{s}_{1}^{\mathrm{T}} \mathbf{s}_{2}\right\|}{\left\|\mathbf{s}_{1}\right\|\left\|\mathbf{s}_{2}\right\|}
$$

$s_{12}$ can range from 0 (no overlap) to 1 (complete overlap), respectively. Data processing and analysis was carried out with tailored in-house developed code in MatLab R2017a (MathWorks, Inc., Natick, MA 2017).

FT-IR Measurements. A Bruker Vertex 80v FT-IR spectrometer (Ettlingen, Germany) equipped with a Globar (power levels of $<15 \mu \mathrm{W}$ for $1 \mathrm{~cm}^{-1}$ spectral range ${ }^{25}$ ) and a liquid nitrogen cooled MCT detector $\left(D^{*}=4 \times 10^{10} \mathrm{~cm} \mathrm{~Hz}^{0.5}\right.$ $\mathrm{W}^{-1}$ at $9.2 \mu \mathrm{m}$ ) was used to perform all FT-IR measurements. The samples were manually injected into a flow cell, equipped with two $\mathrm{CaF}_{2}$ windows and an $8 \mu$ m-thick spacer. During measurements, the spectrometer was constantly flushed with dry air for at least $10 \mathrm{~min}$ prior to data acquisition until water vapor absorption was sufficiently constant. Measurements were performed with a spectral resolution of $2.6 \mathrm{~cm}^{-1}$ in doublesided acquisition mode. A Blackman-Harris 3-term apodization function and a zero-filling factor of 2 were used to calculate the final spectra. In total, 266 scans were averaged per spectrum, leading to an acquisition time of $45 \mathrm{~s}$. All measurements were performed at $25^{\circ} \mathrm{C}$. The software package OPUS 7.2 (Bruker, Ettlingen, Germany) was used for evaluation of spectral data. Water vapor absorption bands were subtracted, if required.

\section{RESULTS AND DISCUSSION}

Mid-IR Spectra Recorded with the EC-QCL Setup. IR transmission spectra of proteins with different concentrations in aqueous solutions were recorded by using the developed custom-made EC-QCL setup. The absorbance spectra across the amide I and amide II range were calculated by averaging a total number of 300 scans, enabling qualitative as well as quantitative evaluation. The investigated proteins were selected to exhibit different secondary structures revealing diverse spectral features in the mid-IR spectrum (Figure 2A-C). Hemoglobin (Hemo) is mainly composed of $\alpha$-helical secondary structure ${ }^{33,34}$ and shows the characteristic band maximum for $\alpha$-helices at $1656 \mathrm{~cm}^{-1}$ in the amide I region as well as a narrow band at approximately $1545 \mathrm{~cm}^{-1}$ in the amide II region. ${ }^{35,36} \gamma$-Globulin $(\gamma$-Gl) primarily contains $\beta$-sheet structures resulting in an IR band maximum at $1640 \mathrm{~cm}^{-1}$ in the amide $I$ region and a broad band with a maximum at approximately $1550 \mathrm{~cm}^{-1}$ in the amide II region. ${ }^{37}$ Concanavalin A (ConA) is predominantly composed of two antiparallel $\beta$-sheets ${ }^{38}$ and displays a distinctive IR spectrum with a band maximum at $1636 \mathrm{~cm}^{-1}$ and a sideband at 1694 $\mathrm{cm}^{-1}$ in the amide I region as well as a broad band with a maximum at $1536 \mathrm{~cm}^{-1}$ in the amide II region. ${ }^{7,39,40}$ To highlight the high-quality spectra obtainable at low concentration, Figure 3 depicts an overlay of the QCL-IR spectra recorded at a protein concentration of $0.1 \mathrm{mg} \mathrm{mL}$. The amide I and amide II bands can be clearly distinguished from the noise level. Even though the spectra at such low

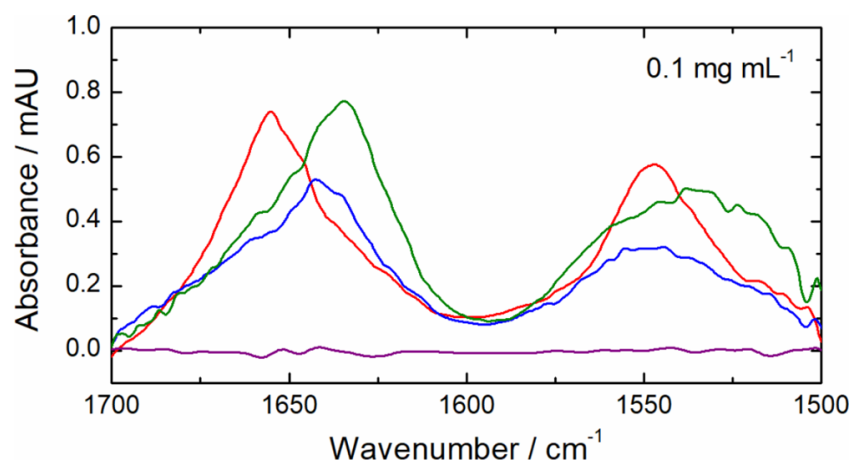

Figure 3. QCL-IR absorbance spectra of hemoglobin (red line), $\gamma$ globulin (blue line), and concanavalin A (green line) at concentration levels of $0.1 \mathrm{mg} \mathrm{mL}^{-1}$. For reference, a $100 \%$ line of water indicates the noise level (purple line).

concentration levels are not completely smooth, the characteristic band shapes of the individual proteins can be clearly identified.

The spectra obtained in the laser-based setup were compared with FT-IR measurements. Figure 2D-F shows the FT-IR spectra of $20 \mathrm{mg} \mathrm{mL}^{-1}$ protein solutions measured at a path length of $8 \mu \mathrm{m}$. Evaluation of the shape and position of the absorbance bands show high comparability between the EC-QCL setup and FT-IR spectroscopy. By using eq 2, the $s_{12}$ values obtained for Hemo, ConA and $\gamma$-Gl were 0.99666, 0.99271 , and 0.99202, respectively, demonstrating excellent comparability of the spectra obtained with the laser-based IR setup and conventional FT-IR spectroscopy.

For evaluation of the capabilities of the developed setup for quantitation purposes, the height of the band maxima in the amide I region was evaluated for protein solutions with eight different concentrations ranging between 0.05 and $10 \mathrm{mg}$ $\mathrm{mL}^{-1}$. The calibration curves in Figure S2 in the Supporting Information demonstrate high linearity $\left(r^{2}>0.999\right)$ down to concentrations of $0.05 \mathrm{mg} \mathrm{mL}$. Hence, the validity of Lambert-Beer's law is demonstrated for the method and allows application of the developed setup for quantitative analysis.

Adaptation of Laser Emission and Detector Characteristics. To achieve sensitive measurements in the laser setup, the SPD on the detector needs to be well within the dynamic range of the MCT detector across the full spectral range of the setup and most similar in both arms of the balancing scheme.

Intense absorption of the $\mathrm{HOH}$-bending band of water near $1640 \mathrm{~cm}^{-1}$ leads to substantial SPD differences across the spectral range reaching the detector and exceed its dynamic range. Consequently, the path length of the transmission cell and the laser current need to be carefully optimized. Because of the principles of QCL emission, reduction of the emission power by decreasing the laser current was only possible to a certain degree, because the laser current affects both the emission power as well as the spectral emission range. Consequently, additional optical components were introduced to adjust the power level. Figure S3 in the Supporting Information shows the emission power before and after attenuation with the employed optical elements as well as the corresponding transmission after attenuation. First, a wire mesh was used to reduce the laser light intensity equally across the entire spectral emission range. Second, to attenuate the SPD of the EC-QCL in the amide II region and at the high 
A

Time / s

B
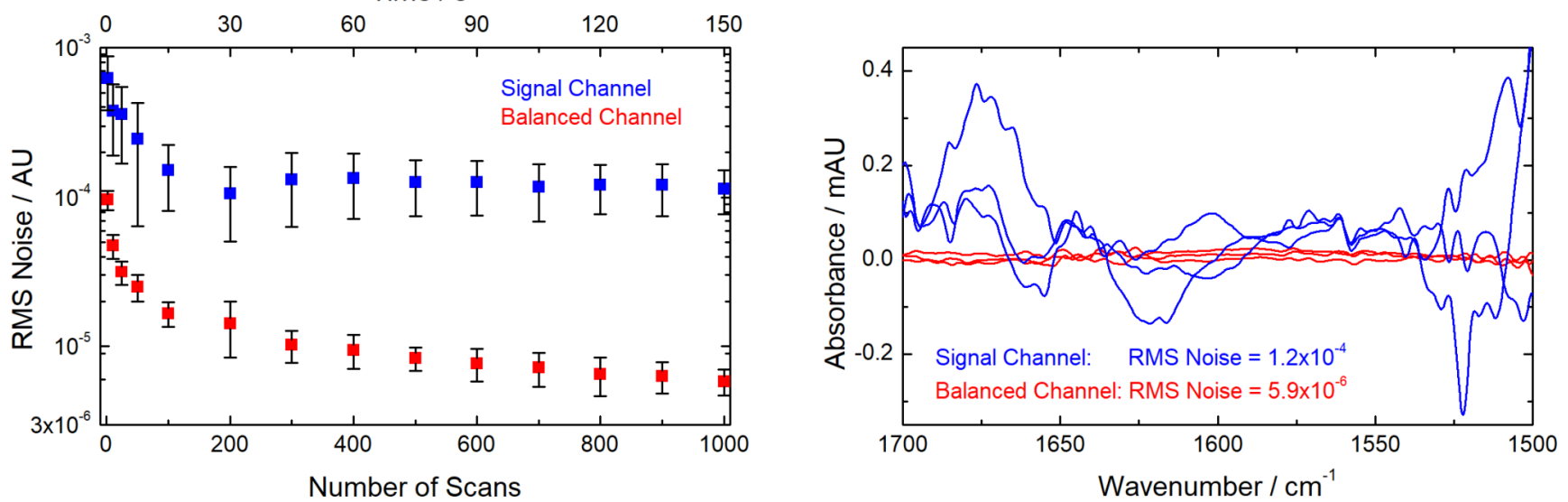

Figure 4. Comparison of the noise level in the signal channel and in the balanced channel of the EC-QCL setup. (A) RMS noise level (average of ten measurements) as a function of time and the total number of scans used for averaging. (B) $100 \%$ lines of water obtained by averaging 1000 scan.

Table 1. Performance Parameters for Comparison of EC-QCL-Based IR Transmission Setups and High-End FT-IR Spectroscopy

\begin{tabular}{|c|c|c|c|c|c|c|}
\hline & meas. time $[\mathrm{s}] /$ scans & $\begin{array}{l}\text { RMS-noise } \\
10^{-5}[\mathrm{AU}]\end{array}$ & $\begin{array}{l}\text { path length } \\
\qquad[\mu \mathrm{m}]\end{array}$ & $\begin{array}{c}\mathrm{LOD} \\
{[\mathrm{mg} / \mathrm{mL}]}\end{array}$ & $\begin{array}{c}\text { spectral range } \\
{\left[\mathrm{cm}^{-1}\right]}\end{array}$ & $\begin{array}{c}\text { detector type/temp. } \\
{\left[{ }^{\circ} \mathrm{C}\right] / \text { cooling }}\end{array}$ \\
\hline QCL setup 1st gen. & $250 / 100$ & 37 & 38 & 0.117 & $1700-1600$ & $\mathrm{MCT} /-60 / \mathrm{TE}$ \\
\hline QCL setup 2nd gen. & $53 / 100$ & 6.2 & 31 & 0.025 & $1700-1500$ & $\mathrm{MCT} /-78 / \mathrm{TE}$ \\
\hline QCL balanced setup & $45 / 300$ & 1.0 & 26 & 0.0043 & $1700-1500$ & $\mathrm{MCT} /-73 / \mathrm{TE}$ \\
\hline high-end FT-IR & $45 / 266$ & 2.3 & 8 & 0.035 & $4000-600$ & $\mathrm{MCT} /-196 / \mathrm{LN}_{2}$ \\
\hline
\end{tabular}

wavenumber end of the amide I band, but to preserve high emission powers near the region of high IR absorption of water, wavelength selective filters were employed. A wedged sapphire window was used as a short-wave pass filter to reduce the laser intensity in the amide II region. For reducing the laser intensity at the high wavenumber side of the amide I band, a silicon-based long-wave pass filter was applied. This attenuation of the beam combined with laser current optimization and an optimized path length of $26 \mu \mathrm{m}$ led to SPD across the entire amide I and amide II region that was well within the dynamic range of the detector.

Balanced Detection for Reduction of Spectral Noise. In the presented EC-QCL setup, a novel balanced MCT module was employed. This detector provides individual outputs for the intensity levels of the two beam paths as well as the balanced channel, which corresponds to the difference between the two channels. This balanced detection approach enables to reduce the intensity noise inherent in the laser emission spectrum. To achieve maximum noise suppression in the balanced channel, the received power in the two channels should be as equal as possible. As opposed to the work of Kübel et al., ${ }^{29}$ who used an aperture to adjust the intensity of the reference beam, we used two almost identical flow cells, filled with sample and solvent, for achieving the most similar intensities across the whole tuning range for both channels. Variations in the splitting ratio of the beam splitter across the tuning range of the laser as well as differences in the optical path length of both transmission cells require additional fine adjustment of one of the two beam paths to fulfill this criterion. For this purpose, a $\mathrm{CaF}_{2}$ window was used to specifically attenuate the light intensity in one of the two beams. For typical signals (reference, signal, balanced), see Figure S4 in the Supporting Information.
The noise level of the EC-QCL setup was determined using $100 \%$ lines from the absorbance spectra of two consecutively recorded measurements with water in both transmission cells. Ideally, the spectrum would reveal a flat line at zero absorbance corresponding to $100 \%$ transmittance. ${ }^{41}$ The RMS (rootmean-square) noise was calculated between 1700 and 1600 $\mathrm{cm}^{-1}$. Figure 4A shows the average noise level as well as the standard deviation of 10 repeated measurements in the signal channel and in the balanced channel as a function of total number of averaged scans. For the signal channel, the lowest noise level of $\sim 1.2 \times 10^{-4} \mathrm{AU}$ was reached by averaging 200 scans. Here, a higher number of scans did not improve the achievable noise level. In contrast, the RMS noise evaluated from balanced channel further decreased with a higher number of scans. Moreover, the variation among repeated measurements is distinctively lower in the balanced channel as indicated by the lower standard deviation (error bars). Finally, by averaging 1000 scans, an average RMS noise of $5.9 \times 10^{-6}$ AU was reached, which is approximately 20 times lower than the noise level in the signal channel (see Figure 4B). This thorough characterization of the noise level enables high flexibility for future applications. The acquisition time can be modified in accordance to the required sensitivity. While an acquisition time of $150 \mathrm{~s}$ can be used, when maximum sensitivity is required, much shorter measurement times are feasible for solutions of high protein content.

Comparison of Setup Performance to FT-IR Spectroscopy and Previous EC-QCL Setups. The noise level of the laser-based balanced detection setup was evaluated and compared with a commercially available high-end FT-IR spectrometer as well as with custom-built IR transmission setups employing first-generation ${ }^{15}$ and state-of-the-art ECQCLs $^{19}$ without balanced detection modules. Performance parameters are summarized in Table 1 . To evaluate the noise 
floor, the RMS noise of $100 \%$ lines of water was calculated in the $1700-1600 \mathrm{~cm}^{-1}$ region. Mechanical imperfections and triggering issues of the first-generation EC-QCL introduced considerable noise levels in the calculated absorbance spectra. Therefore, COW, a rubberband like alignment method, was used to compensate these flaws, leading to an RMS noise of $26.5 \times 10^{-5} \mathrm{AU}$ at significantly longer measurement times due to limited laser sweep rates. For the second-generation ECQCL setup, an adapted data processing approach was employed. Here, evaluation by similarity index was used to remove shifted outlier scans among otherwise highly overlapping scans. Compared with the first-generation setup, a SNR improved by a factor of $\sim 5$ could be achieved within a significantly lower measurement time. For the newly developed balanced detection setup, the measurement time was even further reduced by combining the high sweep rates of the latest generation EC-QCL with a state-of-the-art lock-in amplifier. With this optimized signal-processing scheme, approximately 3.5 times more scans could be recorded during the same acquisition time than in the previously reported setup. ${ }^{19}$

For the herein presented setup, an RMS noise of $1.0 \times 10^{-5}$ AU was achieved at an acquisition time of $45 \mathrm{~s}$ using a path length of $26 \mu \mathrm{m}$. Compared with the previous EC-QCL setups, the slightly lower applicable path length resulted from the trade-off between the path length and the dynamic range of the detector. While the employed data processing routine was the same as for the second-generation setup, the obtained RMS noise was approximately 6.2 times lower within a slightly shorter measurement time. Furthermore, the performance was compared to an FT-IR spectrometer equipped with a fast interferometer and a liquid nitrogen-cooled MCT detector. Previous QCL setups could not reach the low noise levels of this high-end instrument.

To compare the performance, the same spectral resolution and acquisition time were set for recording $100 \%$ lines of water with the laser-based in FT-IR spectrometer. A path length of 8 $\mu \mathrm{m}$ was employed for FT-IR measurements. The final noise level accomplished with the EC-QCL setup was approximately 2.3 times lower than with the high-end FT-IR spectrometer.

Additionally, the limit of detection (LOD) was determined by using

$$
\text { LOD }=\frac{3 \times \text { noise }}{\text { slope of the calibration function }}
$$

Here, not only the RMS noise is considered but also the linear calibration function calculated from the height of the band maxima of protein solutions with different concentration levels. According to Lambert-Beer's law, a higher signal intensity is reached when larger optical path lengths are applied for transmission measurements. Thus, the short path length of 8 $\mu \mathrm{m}$ that has to be used in FT-IR spectroscopy does not only considerably reduce the robustness of the measurement but also impairs its sensitivity. Hence, the superiority of the laserbased setup is even more pronounced in terms of the LOD than in the noise level. When comparing at the same acquisition time of $45 \mathrm{~s}$, the LOD of the FT-IR measurements was 8 times higher than that of the laser-based balanced detection setup. Finally, the LOD of the laser-based setup can be further decreased to $0.0025 \mathrm{mg} \mathrm{mL}^{-1}$ by increasing the measurement time to $150 \mathrm{~s}$. For the same increase in measurement time, the LOD for FT-IR spectroscopy decreases by approximately the same factor to $0.022 \mathrm{mg} \mathrm{mL}^{-1}$.

\section{CONCLUSIONS AND OUTLOOK}

In this work, a new mid-IR transmission setup was introduced for recording protein spectra in the amide I and II region by using an EC-QCL and an MCT balanced detection module. Thorough characterization of the performance revealed noise levels better by a factor of up to 20 in balanced detection mode compared with single-channel measurements. This indicates that the noise introduced by the high-intensity laser light source can be successfully compensated with the balanced detection module. An improved data acquisition scheme allowed recording a high number of scans during short measurement times. The total acquisition time can be adapted in order to fulfill the required sensitivity, allowing high flexibility for IR measurements of proteins. By using an acquisition time of $45 \mathrm{~s}$, characteristic spectral features of proteins with different secondary structures were successfully measured at concentrations as low as $0.1 \mathrm{mg} \mathrm{mL}^{-1}$. Furthermore, quantitative as well as qualitative application of the setup for protein solutions with significantly lower concentration levels is possible when exact secondary structure determination is not required. Compared with high-end FT-IR spectroscopy, the achieved LOD was approximately 8 times lower at similar measurement times. The approach also compares well to other novel, commercially available QCLbased IR spectroscopy systems, such as the AQS3pro (RedShiftBio), which provides a similar performance in terms of LOD and sensitivity to secondary structure at approximately 10 -fold measurement time and a reduced spectral coverage (amide I band only). ${ }^{42}$

The enhanced performance of the presented setup allows application of mid-IR spectroscopy for the analysis of proteins in aqueous solutions at previously prohibitively low concentration levels. Furthermore, the large optical path length of our laser-based approach also allows robust sample handling. Within the covered spectral region, the presented setup outperforms previous EC-QCL-based setups as well as highend FT-IR spectroscopy and marks the next generation of midIR spectroscopy of proteins in aqueous solutions. In the future, this setup will be employed for chemometrics-based protein quantification in complex matrices (e.g., milk) and analysis of bioprocess products, where the protein concentration at certain process steps are below the range accessible by conventional FT-IR spectroscopy.

\section{ASSOCIATED CONTENT}

\section{Supporting Information}

The Supporting Information is available free of charge at https://pubs.acs.org/doi/10.1021/acs.analchem.0c01406.

Working principle of the balanced detection module, calibration curves of proteins, laser emission curve with and without attenuation, single beam spectra of reference, signal and balanced channel (PDF)

\section{AUTHOR INFORMATION}

\section{Corresponding Authors}

Bernhard Lendl - Institute of Chemical Technologies and Analytics, Technische Universität Wien, 1060 Vienna, Austria; ○ orcid.org/0000-0003-3838-5842; Email: bernhard.lendl@ tuwien.ac.at

Andreas Schwaighofer - Institute of Chemical Technologies and Analytics, Technische Universität Wien, 1060 Vienna, 
Austria; @ orcid.org/0000-0003-2714-7056;

Email: andreas.schwaighofer@tuwien.ac.at

\section{Authors}

Christopher K. Akhgar - Institute of Chemical Technologies and Analytics, Technische Universität Wien, 1060 Vienna, Austria; $\odot$ orcid.org/0000-0001-8266-043X

Georg Ramer - Institute of Chemical Technologies and Analytics, Technische Universität Wien, 1060 Vienna, Austria; ๑ orcid.org/0000-0001-8307-5435

Mateusz Żbik - Vigo System S.A., 05-850 Oz̈arów, Mazowiecki, Poland

Artur Trajnerowicz - Vigo System S.A., 05-850 Ö̈arów, Mazowiecki, Poland

Jarosław Pawluczyk - Vigo System S.A., 05-850 Oz̈arów, Mazowiecki, Poland

Complete contact information is available at:

https://pubs.acs.org/10.1021/acs.analchem.0c01406

\section{Notes}

The authors declare no competing financial interest.

\section{ACKNOWLEDGMENTS}

This work has received funding by the European Union's Horizon 2020 research and innovation programme through the NUTRISHIELD project under grant agreement no. 818110 . A.S. gratefully acknowledges financial support by the Austrian Science Fund FWF (project no. P32644-N).

\section{REFERENCES}

(1) Fabian, H.; Mäntele, W. Infrared Spectroscopy of Proteins. In Handbook of Vibrational Spectroscopy; Griffiths, P., Chalmers, J. M., Eds.; John Wiley \& Sons, Ltd.: Hoboken, NJ, 2006.

(2) Murphy, B.; D’Antonio, J.; Manning, M.; Al-Azzam, W. Curr. Pharm. Biotechnol. 2014, 15, 880-889.

(3) Singh, B. R. Basic Aspects of the Technique and Applications of Infrared Spectroscopy of Peptides and Proteins. Infrared Analysis of Peptides and Proteins; ACS Symposium Series; American Chemical Society: Washington, DC, 1999; pp 2-37.

(4) Srour, B.; Brüchert, S.; Andrade, S.; Hellwig, P. Secondary Structure Determination by Means of ATR-FTIR Spectroscopy. In Membrane Protein Structure and Function Characterization: Methods and Protocols; Lacapere, J.-J., Ed.; Springer New York: New York, NY, 2017; pp 195-203.

(5) Wilcox, K. E.; Blanch, E. W.; Doig, A. J. Biochemistry 2016, 55, 3794-3802.

(6) Shariati-Rad, M.; Hasani, M. Biochimie 2009, 91, 850-856.

(7) Dousseau, F.; Pezolet, M. Biochemistry 1990, 29, 8771-8779.

(8) Navea, S.; Tauler, R.; Goormaghtigh, E.; De Juan, A. Proteins: Struct., Funct., Genet. 2006, 63, 527-541.

(9) Alcaraz, M. R.; Schwaighofer, A.; Goicoechea, H.; Lendl, B. Spectrochim. Acta, Part A 2017, 185, 304-309.

(10) Cooper, E. A.; Knutson, K. Fourier Transform Infrared Spectroscopy Investigations of Protein Structure. In Physical Methods to Characterize Pharmaceutical Proteins; Herron, J. N., Jiskoot, W., Crommelin, D. J. A., Eds.; Springer US: Boston, MA, 1995; pp 101143.

(11) Kong, J.; Yu, S. Acta Biochim. Biophys. Sin. 2007, 39, 549-559.

(12) Yang, H.; Yang, S.; Kong, J.; Dong, A.; Yu, S. Nat. Protoc. 2015, 10, 382-396.

(13) Faist, J.; Capasso, F.; Sivco, D.; Sirtori, C.; Hutchinson, A.; Cho, A. Science 1994, 264, 553-556.

(14) Schwaighofer, A.; Brandstetter, M.; Lendl, B. Chem. Soc. Rev. 2017, 46, 5903-5924.
(15) Alcaraz, M. R.; Schwaighofer, A.; Kristament, C.; Ramer, G.; Brandstetter, M.; Goicoechea, H.; Lendl, B. Anal. Chem. 2015, 87, 6980-6987.

(16) Alcaraz, M. R.; Schwaighofer, A.; Goicoechea, H.; Lendl, B. Anal. Bioanal. Chem. 2016, 408, 3933-3941.

(17) Schwaighofer, A.; Alcaraz, M. R.; Araman, C.; Goicoechea, H.; Lendl, B. External cavity-quantum cascade laser infrared spectroscopy for secondary structure analysis of proteins at low concentrations. Sci. Rep. 2016, 6.

(18) Kuligowski, J.; Schwaighofer, A.; Alcaraz, M. R.; Quintas, G.; Mayer, H.; Vento, M.; Lendl, B. Anal. Chim. Acta 2017, 963, 99-105.

(19) Schwaighofer, A.; Montemurro, M.; Freitag, S.; Kristament, C.; Culzoni, M. J.; Lendl, B. Anal. Chem. 2018, 90, 7072-7079.

(20) Schwaighofer, A.; Kuligowski, J.; Quintas, G.; Mayer, H. K.; Lendl, B. Food Chem. 2018, 252, 22-27.

(21) Montemurro, M.; Schwaighofer, A.; Schmidt, A.; Culzoni, M. J.; Mayer, H. K.; Lendl, B. Analyst 2019, 144, 5571-5579.

(22) Schwaighofer, A.; Alcaraz, M. R.; Lux, L.; Lendl, B. pH titration of beta-lactoglobulin monitored by laser-based Mid-IR transmission spectroscopy coupled to chemometric analysis. Spectrochim. Acta, Part A 2020, 226.117636

(23) Schwaighofer, A.; Lendl, B. Chapter 3 - Quantum cascade laserbased infrared transmission spectroscopy of proteins in solution. In Vibrational Spectroscopy in Protein Research; Academic Press, 2020; pp 59-88.

(24) Hayden, J.; Hugger, S.; Fuchs, F.; Lendl, B. A quantum cascade laser-based Mach-Zehnder interferometer for chemical sensing employing molecular absorption and dispersion. Appl. Phys. B: Lasers Opt. 2018, 124.29

(25) Brandstetter, M.; Koch, C.; Genner, A.; Lendl, B. Proc. SPIE 2014, 8993, 89931U.

(26) Hobbs, P. Shot noise limited optical measurements at baseband with noisy lasers (proceedings Only). Proc. SPIE 1990, 1376, Laser Noise. 216

(27) Waclawek, J. P.; Kristament, C.; Moser, H.; Lendl, B. Opt. Express 2019, 27, 12183-12195.

(28) Reyes-Reyes, A.; Hou, Z.; van Mastrigt, E.; Horsten, R. C.; de Jongste, J. C.; Pijnenburg, M. W.; Urbach, H. P.; Bhattacharya, N. Opt. Express 2014, 22, 18299-18309.

(29) Kübel, J.; Botha, C.; Bucka, A.; Höpfner, J.; Zimmermann, H.; Godejohann, M.; Wilhelm, M. Macromol. Rapid Commun. 2019, 40, 1900228.

(30) Lendl, B.; Frank, J.; Schindler, R.; Muller, A.; Beck, M.; Faist, J. Anal. Chem. 2000, 72, 1645-1648.

(31) Edelmann, A.; Ruzicka, C.; Frank, J.; Lendl, B.; Schrenk, W.; Gornik, E.; Strasser, G. J. Chromatogr. A 2001, 934, 123-128.

(32) Culzoni, M. J.; Goicoechea, H. C.; Ibanez, G. A.; Lozano, V. A.; Marsili, N. R.; Olivieri, A. C.; Pagani, A. P. Anal. Chim. Acta 2008, 614, 46-57.

(33) Levitt, M.; Greer, J. J. Mol. Biol. 1977, 114, 181-239.

(34) Perutz, M. F.; Rossmann, M. G.; Cullis, A. F.; Muirhead, H.; Will, G.; North, A. C. T. Nature 1960, 185, 416-422.

(35) Barth, A. Biochim. Biophys. Acta, Bioenerg. 2007, 1767, 10731101.

(36) Pan, T.; Peng, A.; Huang, W. Appl. Mech. Mater. 2011, 55-57, $1168-1171$

(37) van de Weert, M.; Haris, P. I.; Hennink, W. E.; Crommelin, D. J. A. Anal. Biochem. 2001, 297, 160-169.

(38) Becker, J. W.; Reeke, G. N., Jr; Wang, J. L.; Cunningham, B. A.; Edelman, G. M. J. Biol. Chem. 1975, 250, 1513-1524.

(39) Alvarez, J.; Haris, P. I.; Lee, D. C.; Chapman, D. Biochim. Biophys. Acta, Protein Struct. Mol. Enzymol. 1987, 916, 5-12.

(40) Arrondo, J. L. R.; Young, N. M.; Mantsch, H. H. Biochim. Biophys. Acta, Protein Struct. Mol. Enzymol. 1988, 952, 261-268.

(41) Brandstetter, M.; Lendl, B. Sens. Actuators, B 2012, 170, 189195.

(42) Ma, E.; Wang, L.; Kendrick, B. Spectroscopy 2018, 33, 46-52. 